Reticulate acropigmentation of Kitamura is characterized by a network of freckle-like areas of pigmentation which develop on the dorsa of the hands in the first two decades, and may subsequently involve most parts of the body. Several individual cases and families have been reported with features of both Kitamura's disease and reticulate pigmented anomaly of the flexures
(Dowling-Degos disease). Mode of inheritance is primarily autosomal dominant. Such conditions are usually refractory to therapy and reassuring the patient is the best modality that can be offered.

Anupam Das, Dipti Das and Anupama Ghosh Department of Dermatology, Medical College and Hospital, Kolkata, India. anupamdasdr@gmail.com

\section{Unilateral Linear Porokeratosis}

A 12-year-old girl presented with multiple, asymptomatic, annular lesions over right side of upper chest and right forearm for last 5 years. Initially, few small brown coloured papules appeared over the chest which gradually increased in number and size to attain the present status. The lesions were asymptomatic from the beginning. There was no history of similar episodes in the past or in the family, and no history of any skin lesions at birth. On examination, multiple skin coloured annular plaques $(1.5-2 \mathrm{cms} \times 3-7 \mathrm{cms}$ in size) with a welldemarcated raised, thready margin with central hypopigmentation and atrophy were found. They were arranged in a linear configuration and unilaterally over right side of upper chest and right forearm (Fig.1 and 2). The lesions had a tendency of peripheral extension and central clearing. Mucosa, scalp and nails were spared. Biopsy was done from one of the lesions and on histopathological examination, hyperkeratosis, cornoid lamella with perivascular dermal infiltrates were seen and central part of the lesion showed atrophy. Biopsy findings confirmed it to be a case of "unilateral linear porokeratosis". She has been prescribed topical retinoids and she is under regular follow-up because this variant of porokeratosis is highly prone to develop malignancy.

Porokeratosis is a disorder of keratinisation, characterised by hyperkeratotic papules or plaques surrounded by a thready elevated border that expands centrifugally. Most cases are sporadic. Pathogenesis is unclear. Seven varities are described. Linear type presents in early childhood and has highest potential for developing squamous cell carcinoma. Histopathological examination gives the definitive diagnosis. The differential diagnoses to be considered are inflammatory linear verrucous epidermal nevus (lesions since birth, erythema, scaling, itching present), stage IV of incontinentia pigmenti (earlier age of onset, preceded by vesicular, verrucous, hyperpigmented stage, associated CNS, dental and ocular defect), linear lichen planus (hyperkeratotic, violaceous, pruritic papule and plaque).

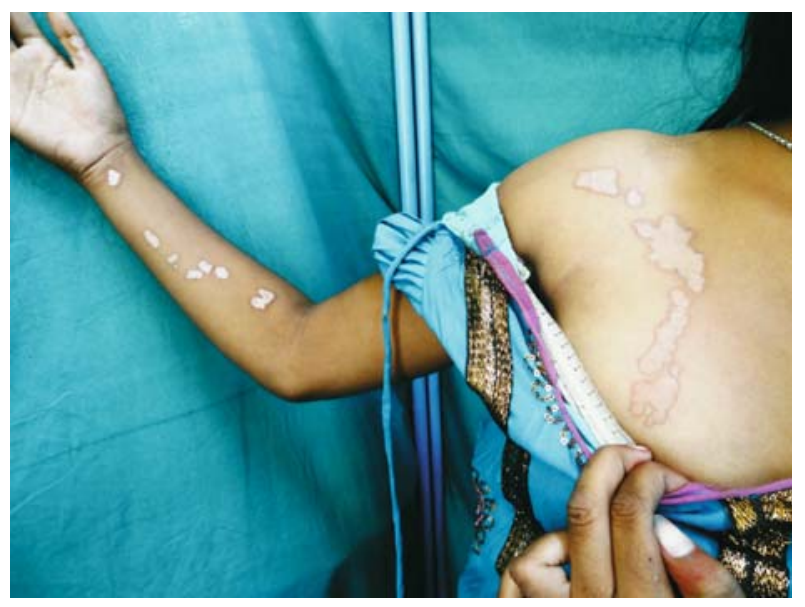

FIG. 1 Linear arrangement of multiple annular plaques.

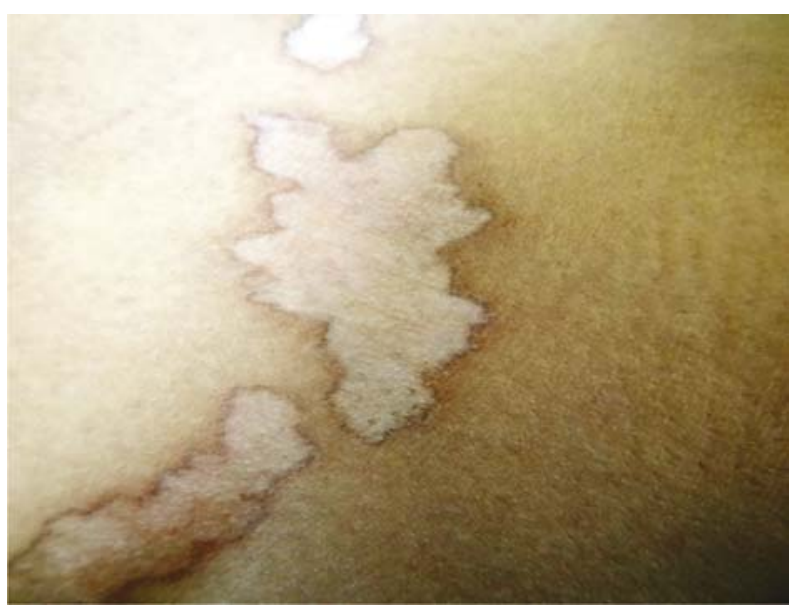

FIG. 2 Skin lesions showing demarcated raised irregular margins with central hypopigmentation.

On histopathology none of them shows cornoid lamella. Topical 5 fluorouracil, topical calcipotriol, topical retinoid, cryotherapy and surgical excision have been tried with various degree of success.

Dipti Das, Anupama Ghosh and Anupam Das Department of Dermatology, Medical College and Hospital, Kolkata, WB, India. anupamdasdr@gmail.com 